\title{
THE EFFECT OF NON-EQUILIBRIUM BOUNDARY LAYERS ON COMPRESSOR PERFORMANCE
}

\author{
Andrew P.S. Wheeler \\ Whittle Laboratory \\ Department of Engineering \\ Cambridge University \\ Cambridge, UK \\ Email: aw329@cam.ac.uk
}

\author{
Anthony M. J. Dickens \\ Whittle Laboratory \\ Department of Engineering \\ Cambridge University \\ Cambridge, UK
}

\author{
Robert J. Miller \\ Whittle Laboratory \\ Department of Engineering \\ Cambridge University \\ Cambridge, UK
}

\begin{abstract}
The paper investigates the effect of non-equilibrium behaviour of boundary layers on the profile loss of a compressor. The investigation is undertaken using both high fidelity simulations of a mid-height section of a compressor blade and a reduced order model, MISES. The solutions are validated using experimental measurements made in the embedded stage of a multistage low speed compressor. The paper shows that up to $35 \%$ of the suction surface boundary layer of the compressor blade exhibits non-equilibrium behaviour. The size of this region reduces as the Reynolds number is increased. The non-equilibrium behaviour was found to reduce profile loss in most cases, however, in a range of cases where transition occurs through a small separation the presence of non-equilibrium behaviour was found to increase profile loss.
\end{abstract}

\section{NOMENCLATURE}

$c \quad$ chord

$C_{a x} \quad$ Axial chord

$c_{d} \quad$ Dissipation coefficient

$c_{f} \quad$ Skin friction coefficient

$C_{p} \quad$ Pressure coefficient

$C_{p o} \quad$ Total pressure coefficient

$c_{\tau} \quad$ Reynolds stress coefficient

$H \quad$ Shape factor

$k \quad$ Turbulent kinetic energy

$M \quad$ Mach number

$p \quad$ Pressure

$\mathrm{Pr} \quad$ Turbulence production rate

$p_{\circ} \quad$ Stagnation pressure

Re Reynolds number based on inlet conditions and chord

$s \quad$ Entropy, surface distance

$\dot{S}_{a} \quad$ Entropy generation rate per unit surface area

$T \quad$ Temperature

$T_{s} \quad$ Isentropic temperature

$T_{\circ} \quad$ Stagnation temperature

Tu Turbulence intensity

$\begin{array}{ll}t & \text { Time } \\ U_{e} & \text { Boundary layer edge velocity } \\ \vec{V} & \text { Velocity vector } \\ V_{i n} & \text { Inlet velocity } \\ x & \text { Axial distance }\end{array}$

$\begin{array}{ll}\text { GREEK LETTERS } & \\ \alpha & \text { Flow angle } \\ \delta & \text { Boundary layer thickness } \\ \delta^{*} & \text { Displacement thickness } \\ \delta_{e} & \text { Energy thickness } \\ \theta & \text { Momentum thickness } \\ \rho & \text { Density } \\ \zeta & \text { Loss coefficient }\end{array}$

\section{INTRODUCTION}

In an aero-engine compressor approximately $40 \%$ of the losses are caused by profile loss [1]. The profile loss is largely determined by the boundary layer development over the blade surface, which creates entropy through viscous shear work. Denton (1993) [2] shows that for a boundary layer, the entropy production rate per unit surface area is

$$
\dot{S}_{a}=c_{d} \frac{\rho U_{e}^{3}}{T}
$$

where the dissipation coefficient $c_{d}$ is a function of the Reynolds number and boundary layer state; typically the dissipation coefficient for laminar boundary layers can be between $2-5$ times lower than for a turbulent one. Therefore, maintaining laminar flow over a significant portion of the blade surface can lead to large reductions in loss. It is largely for this reason and the need to avoid boundary-layer separation, that there have been many studies of boundary-layer transition in compressors blade-rows (see [3-6]). 

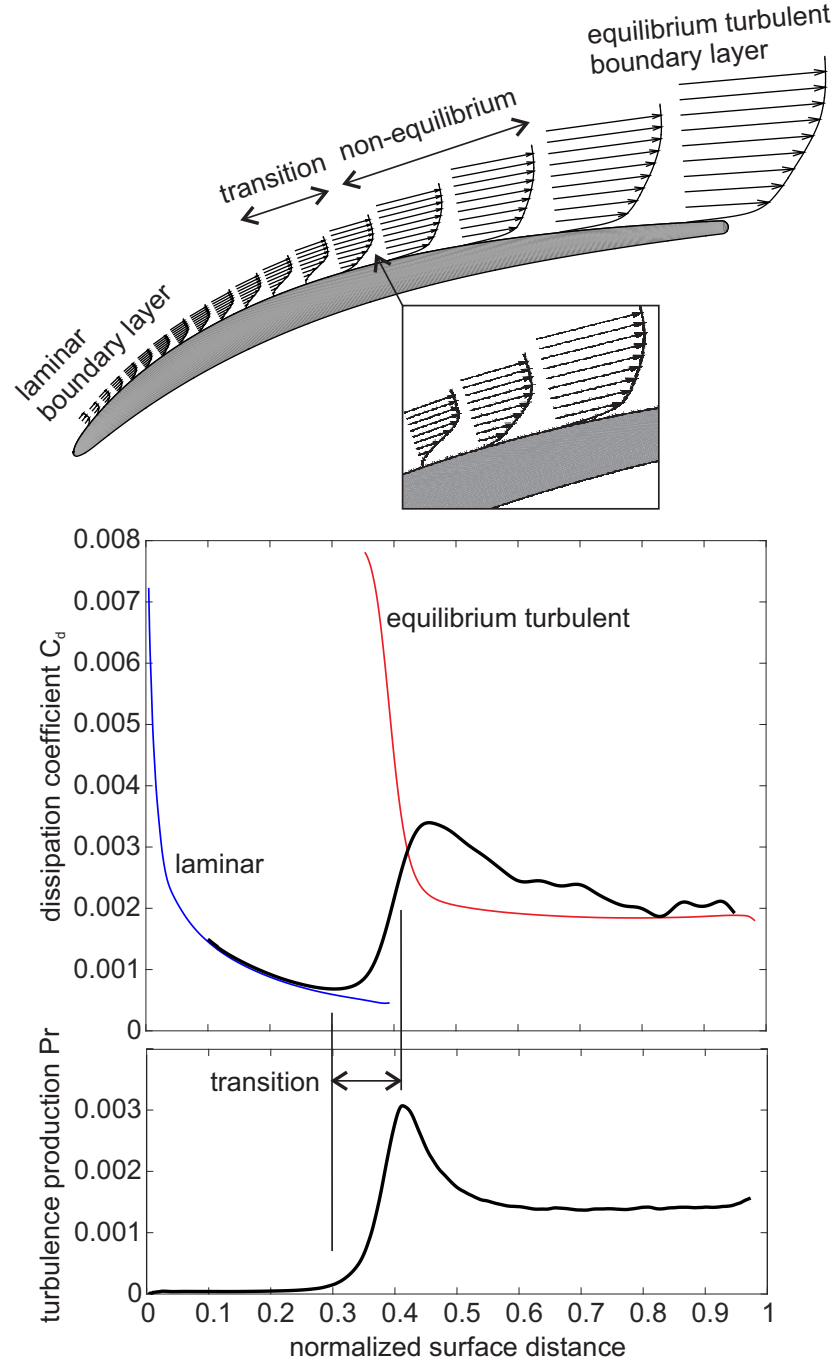

FIGURE 1. Schematic of boundary layer states on a compressor blade suction surface.

The view promoted by equation 1 is that the dissipation coefficient $c_{d}$ is to a large extent only dependent on the boundary layer state, and that loss is determined by the amounts of laminar and turbulent wetted area and loading distribution. Underlying this is the impression that boundary layers exist in two equilibrium states, either laminar or turbulent. A turbulent boundary layer in equilibrium is a boundary layer where the shape of the boundary layer does not vary in the streamwise direction [7]. In reality turbulent boundary layers on compressor blades can behave in a significantly non-equilibrium way. Examples of this include attached boundary layers undergoing transition and reattaching boundary layers. The central aim of this paper is to determine the effect that these non-equilibrium processes can have on profile loss.

In order to illustrate this, Figure 1 shows the suction surface boundary layer states found in the compressor blade studied in this paper as well as the variation in dissipation coefficient and turbulent production. Here we discuss only the suction surface, since this is of most importance to loss. Figure 1 has been put together using data from simulations discussed later in the paper. The dissipation coefficient $c_{d}$ and turbulence production $\operatorname{Pr}$ are determined from the computed entropy generation rate and turbulent shear work integrated across the boundary layer. Across the transition region turbulence production rises, reaches a peak where the flow becomes fully turbulent and then falls back to an equilibrium level over the aft portion of the boundary layer. The dissipation also rises across the transition region, but lags the production such that downstream of transition there is a region where the dissipation and production of turbulence are out of balance; the lag arises because there is a delay between turbulent structures forming and dissipating. This is the physical mechanism which leads to a non-equilibrium turbulent boundary layer state.

Also shown are the dissipation coefficient for laminar and equilibrium turbulent boundary layers as determined by correlations using the local shape factor $H$ and momentum thickness Reynolds number $\operatorname{Re}_{\theta}$ computed from the simulations [8]. The equilibrium dissipation coefficient which is initially very high at transition, rapidly drops to the normal level for attached turbulent boundary layers $\left(c_{d}=0.002\right)$. There is an appreciable difference in the actual dissipation coefficient and the equilibrium values due to the lag between turbulence production and dissipation. In this paper we show that this is important, because the lag means that the peak dissipation is reduced compared to the peak equilibrium level, which tends to reduce overall losses.

In this paper we aim to answer three questions:

1. What percentage of the turbulent boundary layer behaves in a non-equilibrium way?

2. How accurate are reduced order models (shear-lag models) at predicting the non-equilibrium behaviour of compressor boundary layers?

3. What is the impact of non-equilibrium behaviour on profile loss?

These questions are answered using a combination of high fidelity simulations and reduced-order modelling. In the first part of the paper we describe the high-order computational method used. We then describe implicit large eddy and direct numerical simulations of a high-pressure compressor stator passage with freestream turbulence. The simulations are first compared with experimental measurements within a 3-stage compressor in order to ensure that representative free-stream turbulence is prescribed and that the predicted total pressure loss matches experimental data. The simulations are used to determine the amount of the stator blade suction surface subjected to non-equilibrium effects and the dependence of this on Reynolds number. The simulations are then used to validate a reduced-order model of nonequilibrium turbulence production using the standard design tool MISES. Finally, this model is used to estimate the extent to which non-equilibrium effects influence loss.

\section{COMPUTATIONAL DETAILS Solver details}

A new high-order code ' $3 D N S$ ' is used for this investigation. $3 D N S$ is a compressible finite-difference Navier-Stokes solver. The algorithm uses explicit differencing with a choice of schemes including standard 4th order and 6th order schemes, and the Tam and Webb [10] 7-point stencil DRP scheme. 


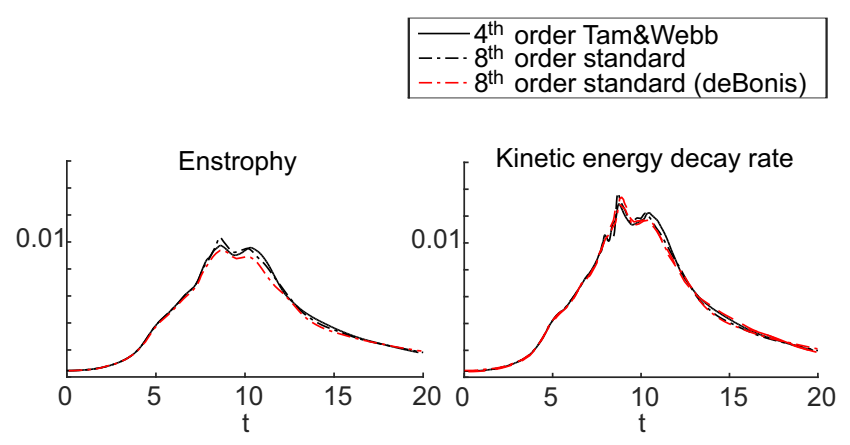

FIGURE 2. Comparison of enstrophy and kinetic energy decay rate against time from the the Taylor-Green vortex case results of de Bonis [9] (red) with $3 D N S$ code using $4^{\text {th }}$ order Tamm and Webb (-) and $8^{\text {th }}$ order (-- ) discretizations with $128^{3}$ mesh points

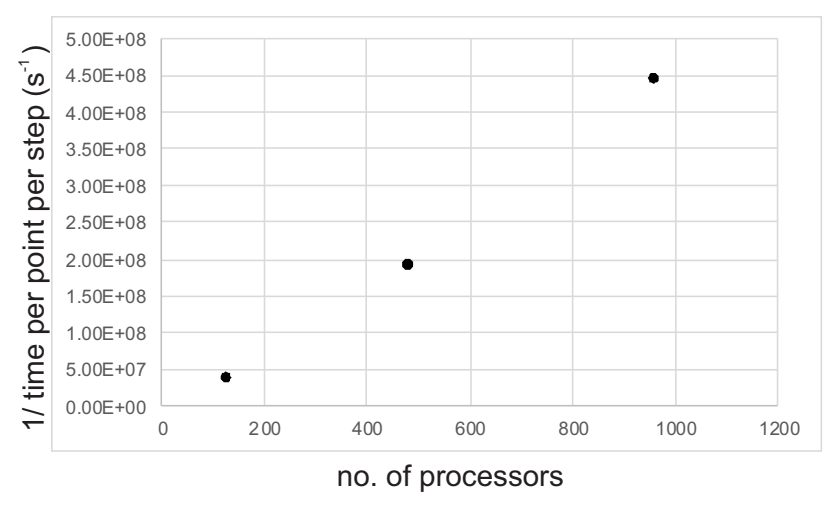

FIGURE 3. Code performance on the Re3E5 case (see table 1)

TABLE 1. Test cases for Reynolds number study

\begin{tabular}{lcccc}
\hline \hline Test-case label & Mesh (no. points) & $R e$ & $T u$ & Span/C $a x$ \\
\hline Re4E5F & Fine $(127 \mathrm{M})$ & $408 \mathrm{k}$ & $3.5 \%$ & 0.1 \\
Re3E5 & Datum $(63 \mathrm{M})$ & $340 \mathrm{k}$ & $3.5 \%$ & 0.1 \\
Re2E5 & Datum $(63 \mathrm{M})$ & $220 \mathrm{k}$ & $3.5 \%$ & 0.1 \\
Re1E5 & Datum $(63 \mathrm{M})$ & $110 \mathrm{k}$ & $3.5 \%$ & 0.1 \\
Re1E5W & Datum (63M) & $110 \mathrm{k}$ & $3.5 \%$ & 0.2 \\
\hline \hline
\end{tabular}

Summation-by-parts boundary schemes are used for the differencing and filtering schemes. The code solves the flow on structured grids which are curvilinear in the blade-to-blade plane, and uniform in the spanwise direction. Time integration is performed using a standard low-storage 4-step Runge-Kutta scheme. The code is multi-block and parallelization is achieved within each block. Characteristic boundary-conditions are applied at both exit and inlet; the method of Poinsot and Lele [11] is implemented. For the case where inflow turbulence is prescribed, the rates of change of velocity are imposed by imposing these on the characteristic variables.

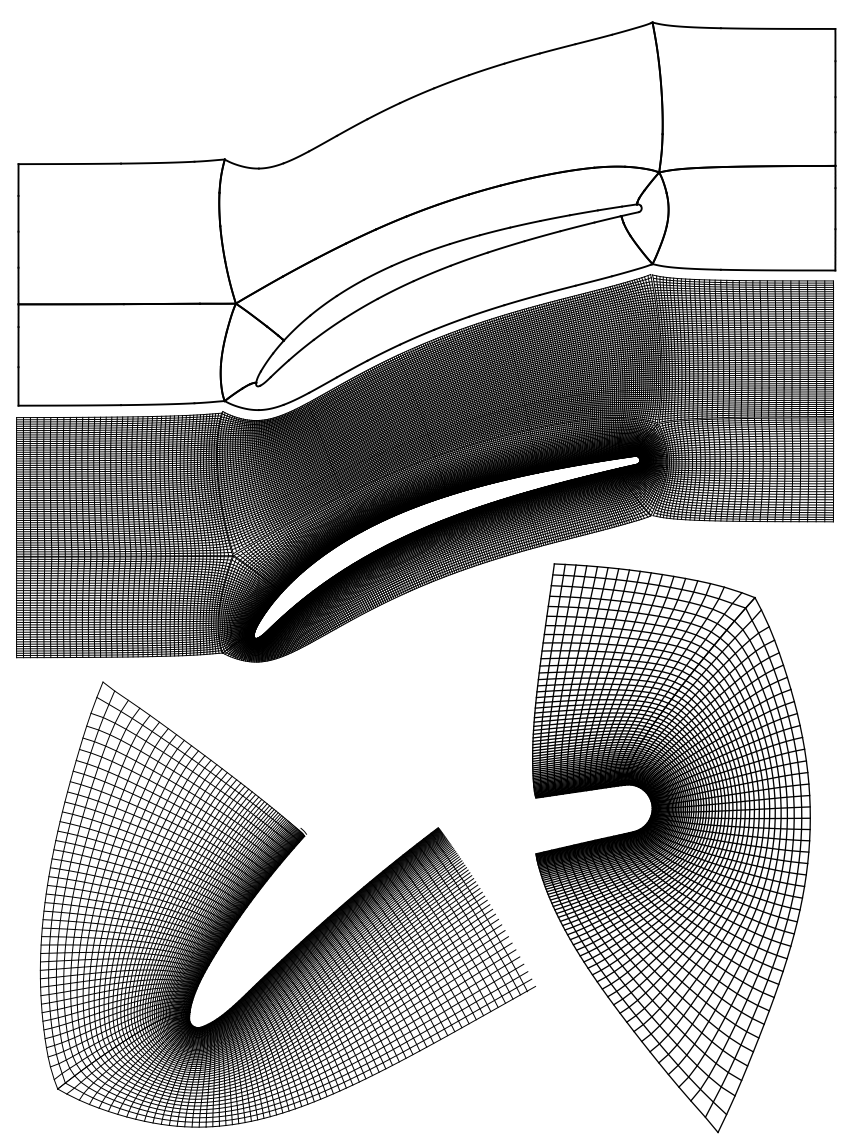

FIGURE 4. Block structure (upper) and example mesh (lower) showing every $4^{\text {th }}$ grid line with leading-edge and trailing-edge detail also showing every $4^{\text {th }}$ grid line ('datum' mesh)

The accuracy of the code has been verified by comparing results for the Taylor-Green Vortex case described by de Bonis [9], as shown in Figure 2. For this investigation the Tam and Webb scheme was used in combination with a standard 8th order filter. Figure 2 shows that this scheme has a comparable accuracy to a standard 8th order scheme.

Strong scaling performance of the code is shown in Figure 3; this shows a linear speed-up in the compute time per point for a $63 M$ point multi-block mesh up to 1000 cores, which is the maximum number of cores used per case for the current investigation.

A description of the test-cases is given in Table 1. These will be described in more detail later. The focus of this work will be to simulate the stator passage flow at mid-span of the SMURF multi-stage compressor rig (described later). A description of the test-cases used for mesh sensitivity checks is given in Table 2 and these are discussed in the following section.

\section{Computational domain and mesh}

The computational domain and example mesh showing the multi-block structure are shown in Figure 4. The geometry is taken from a midspan stator profile (discussed later) and extruded in the spanwise direction to generate a three dimensional domain. A spanwise domain height of $10 \%$ of axial chord was used throughout this study and periodicity was enforced in the 


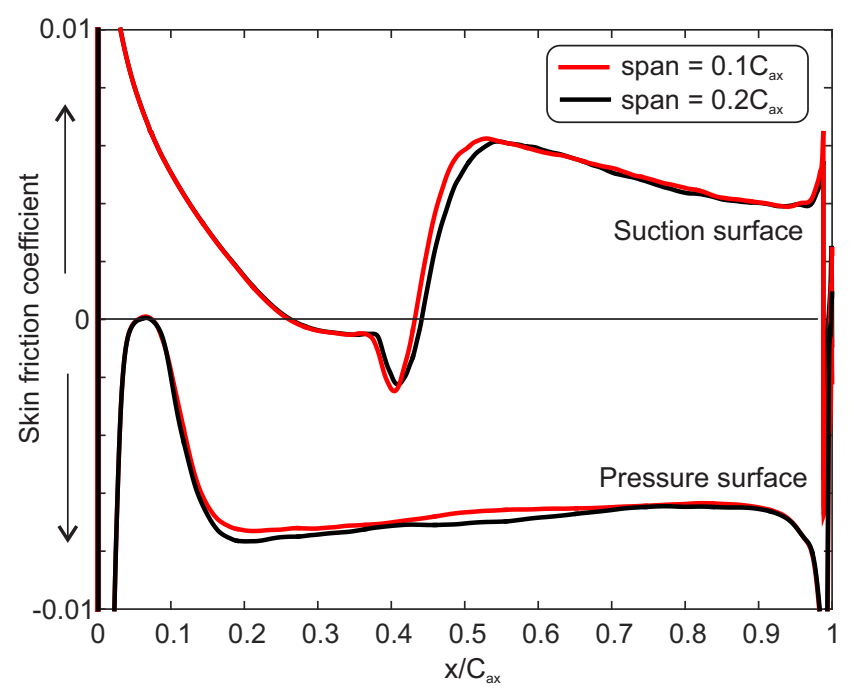

FIGURE 5. The effect of spanwise extent on predicted wall shear stress (cases RE1E5, RE1E5W)

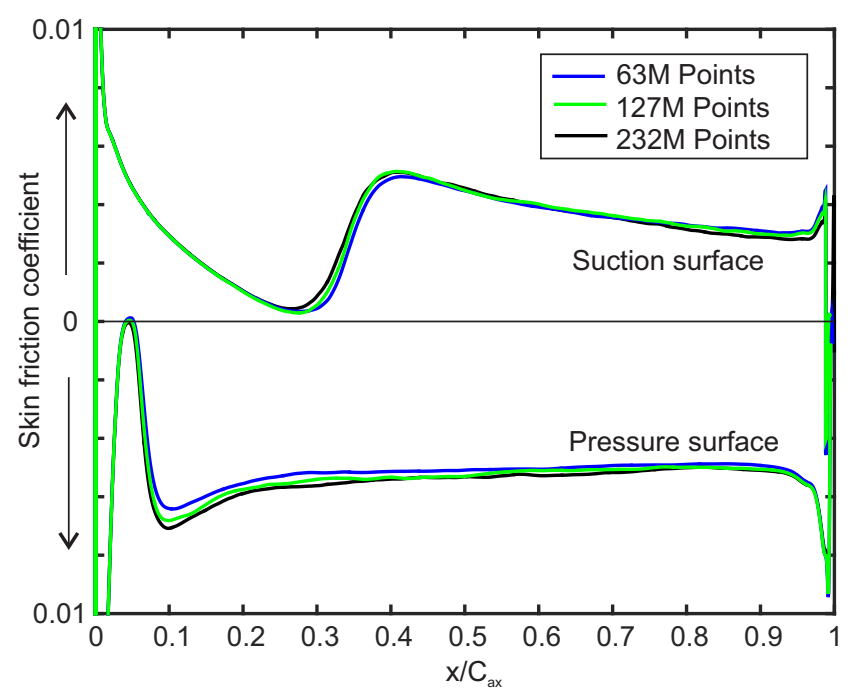

FIGURE 6. Comparison of predicted wall shear stress for three mesh sizes (cases RE3E5, RE3E5F and RE3E5F2)

spanwise direction. The effect of spanwise extent was tested by performing an additional testcase with a span of $20 \%$ of axial chord at $\operatorname{Re}=110 \mathrm{k}$; the results showed only small differences in the skin friction in the region of suction-surface transition (see Figure 5). Meshes were created using a combination of different software. Initially a coarse grid was created using the Turbogrid software from ANSYS. This provided the block structure and block boundaries. A series of codes written in Matlab were then used to construct a much finer grid, ensuring optimum load balancing and near wall mesh control. The majority of the results described in this paper were obtained using a mesh with 63 million points (490k points in the blade-to-blade plane and 128 spanwise points); this will be referred to as the 'datum' mesh. Two finer meshes were also used and referred to as 'fine' and 'fine2'. These had around 25\% and 50\% more cells in each grid direction compared to the datum mesh, giving meshes of $127 \mathrm{M}$ and $240 M$ points respectively. The mesh details and near-wall

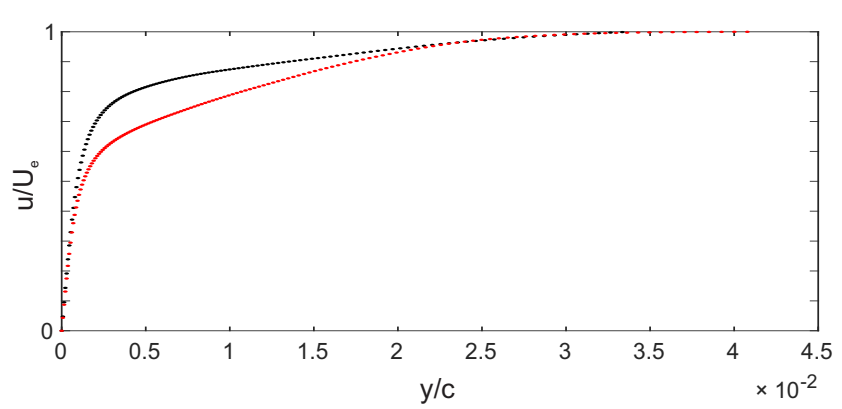

FIGURE 7. Example boundary-layer profiles $\left(x / C_{a x}=0.75\right)$ on the pressure (black) and suction (red) surfaces, 'datum' mesh $R e=340 \mathrm{k}$, $T u=3.5 \%$.

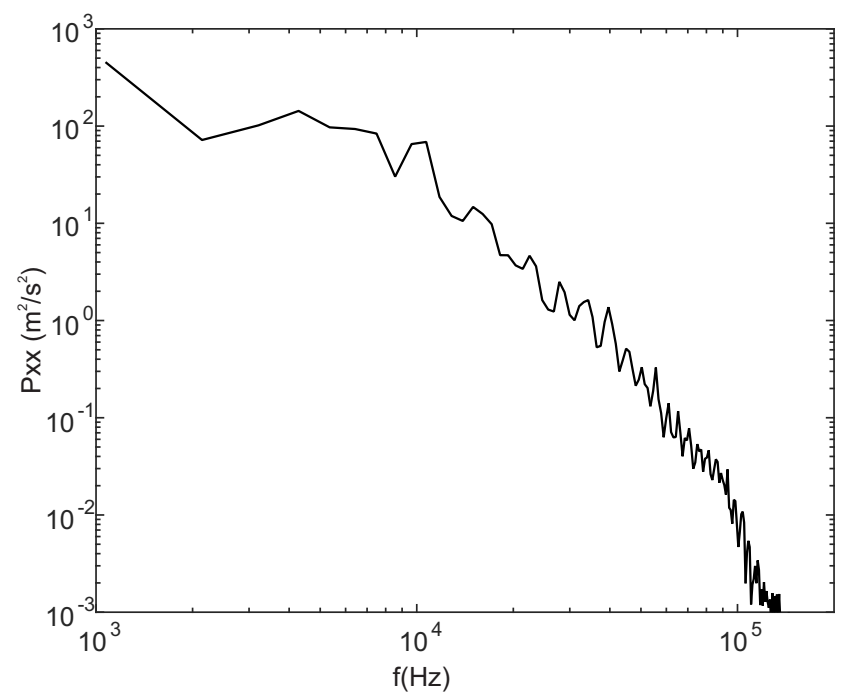

FIGURE 8. Example spectrum within the suction-side boundarylayer $\left(x / C_{a x}=0.98\right.$ and height within boundary layer $\left.y^{+}=30\right)$. 'Datum' mesh $R e=340 k, T u=3.5 \%$.

TABLE 2. Mesh sensitivity test cases and near wall mesh spacing ( $R e=340 k T u=3.5 \%$ )

\begin{tabular}{lcccc}
\hline \hline Test-case label & Mesh (no. points) & $\Delta_{n}^{+}$ & $\Delta_{t}^{+}$ & $\Delta_{z}^{+}$ \\
\hline Re3E5 & Datum (63M) & 1.0 & 18 & 10.5 \\
Re3E5F & Fine (127M) & 1.0 & 14.9 & 8.8 \\
Re3E5F2 & Fine2 (240M) & 1.0 & 12.5 & 7.3 \\
\hline \hline
\end{tabular}

cell sizes are shown in Table 2. The near-wall cell sizes, particularly for the finer meshes, is similar to previous DNS studies such as [12], and so we will refer to the simulations as implicit LES/DNS throughout.

Cell sizes were compared to the Kolmogorov length-scale determined from the computed dissipation; cell sizes for the datum mesh were typically within 5 Kolmogorov length-scales. Previous DNS work (such as [13]) shows that the smallest scales in the flow are typically of the order of 10 Kolmogorov scales 
and grid independence is achieved when cell sizes are around 510 Kolmogorov lengths. Mesh sensitivity was analyzed by comparing results from the $R E 3 E 5$ case which had $63 M$ mesh points with the results for the two finer grids ( $R E 3 E 5 F$ and $R E 3 E 5 F 2$ ). The wall shear stress for these two cases is shown in Figure 6. The figure shows small differences around regions of transition on the suction and pressure surfaces, but in general the three cases are in very close agreement particularly on the suctionsurface which is the main focus of this paper.

Figure 7 shows typical velocity profiles within the turbulent regions of the suction and pressure surfaces. The figure shows around 100 points within each boundary layer. Figure 8 shows an example of the power spectrum obtained in the aft suction surface for the 'datum' mesh, showing that the turbulent kinetic energy is resolved over around $3-4$ orders of magnitude.

\section{Time-averaging}

Time-average and statistical quantities were gathered during the simulations by summing the instantaneous conserved quantities $\left(\rho, \rho u, \rho v, \rho w, E_{t}\right)$ and also momentum terms $\left(\rho u^{2}, \rho v^{2}, \rho w^{2}, \rho u v, \rho u w, \rho v w\right)$. Time-average primitive variables were determined from these; this is equivalent to a Favreaverage for velocity terms. Time-averaging of instantaneous entropy fluxes was also performed $(\rho u s, \rho v s, \rho w s)$ in order to determine loss and entropy generation rates used in the determination of dissipation coefficients. Convergence of the statistics was checked by comparing the computed loss over different averaging times, to ensure the data was statistically stationary; typically, time-averages were computed over a time equivalent to 3 passings of the flow through stator passage.

\section{Inflow turbulence generation}

The inflow turbulence generation makes use of the library generated by Phillips and Fyfe [14] which generates a Gaussian turbulence spectrum. Turbulence fluctuations were precomputed and then introduced at the inflow boundary at runtime. A length-scale and intensity were chosen to match experimental data obtained in a multi-stage compressor test rig as discussed later. A snap-shot of the structure of the free-stream turbulence in the region of the stator leading-edge is shown in Figure 9 which shows iso-surfaces of Q-criterion, which identifies vortical structures in the flow. Figure 10 shows contours of spanwise vorticity for cases with zero free-stream turbulence ('clean') and with $T u=3.5 \%$. For the 'clean' case, on both the pressure and suction surfaces, the flow breaks down to turbulence via an essentially two-dimensional mechanism of Kelvin-Helmholtz roll-ups which eventually become three-dimensionally unstable. The addition of free-stream turbulence causes a much earlier breakdown to turbulence, although separation still occurs on both surfaces.

\section{Matching to rig conditions}

The numerical study is undertaken on the mid-height profile of an embedded low speed compressor stage at the Whittle Laboratory. To ensure that the findings of the numerical study are representative of this stage experimental measurements were

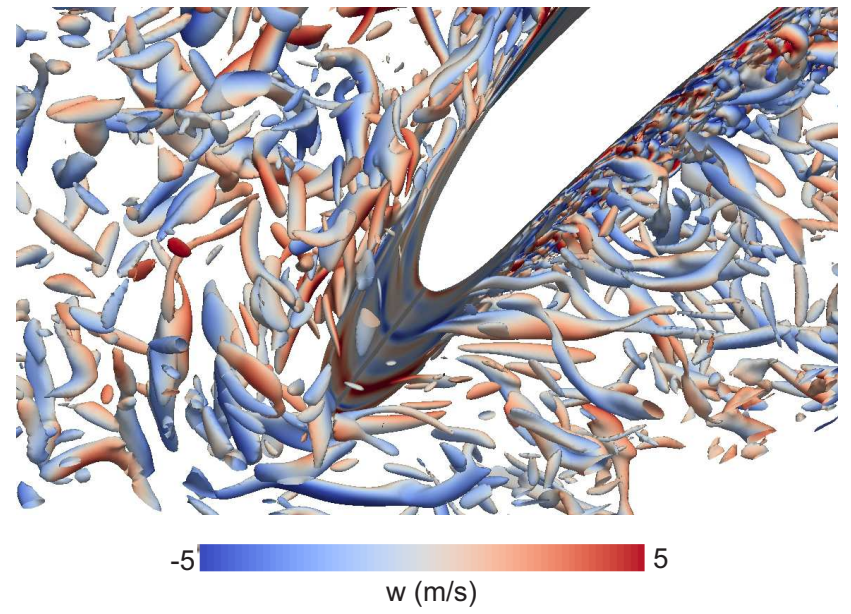

FIGURE 9. Iso-surfaces of Q-criterion $=3 \times 10^{7} s^{-2}$. Colour indicates spanwise velocity

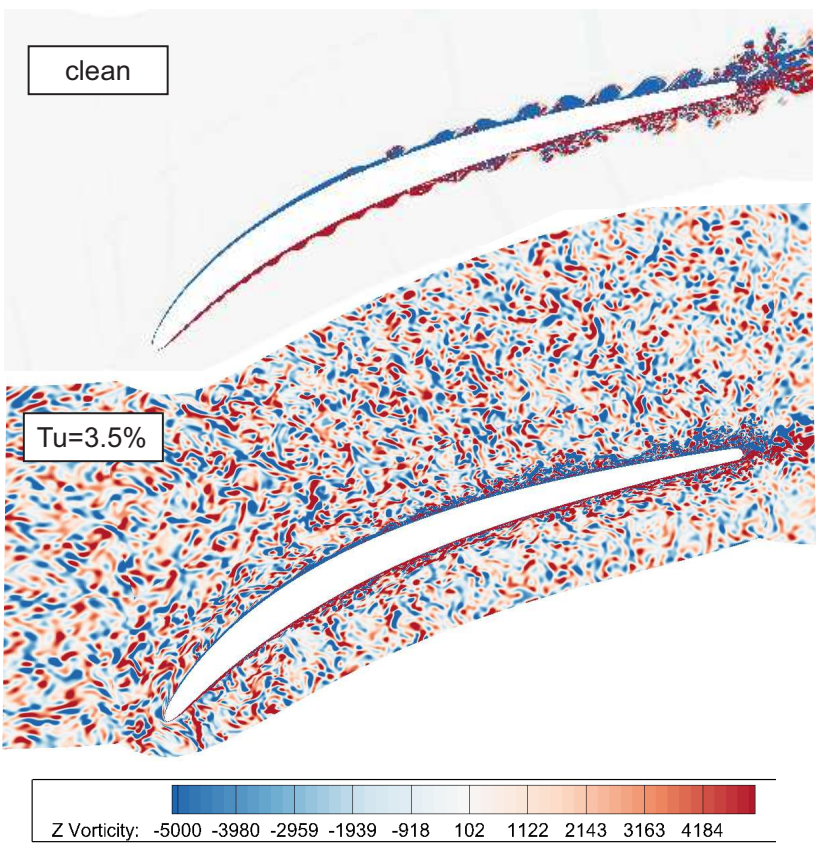

FIGURE 10. Instantaneous spanwise vorticity contours with and without freestream turbulence $(R e=340 k)$

compared with the results from the computational simulations. Firstly, the turbulence level and spectrum at mid-height stator inlet was measured and was used to verify that the inflow turbulence used in the simulations was representative. Secondly, to ensure that the loss was accurately predicted the wake profile was measured and compared against the results of the simulations.

The SMURF Rig (see Figure 11) simulates a multi-stage high-pressure compressor; the rig is a 3 -stage large-scale $(1.5 \mathrm{~m}$ diameter) model, which operates at low Mach number $(M \approx 0.1)$ with cylindrical and parallel endwalls. The simulations were performed to match the stator 3 (S3) design-point conditions; the Reynolds number based on stator inlet conditions and chord is $R e=340 \mathrm{~K}$ and the inlet flow angle is $46^{\circ}$. At these conditions the flow at mid-span of the stator is very closely two-dimensional; 


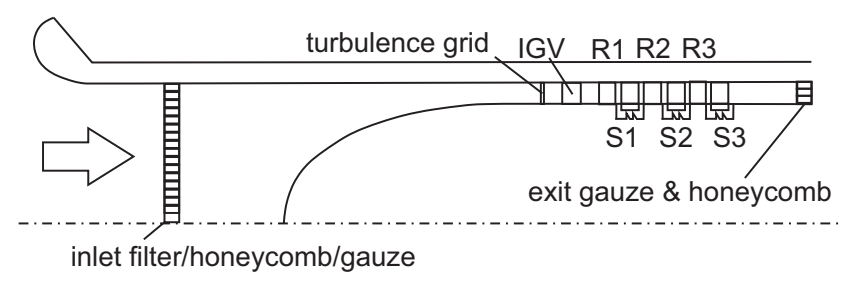

FIGURE 11. Schematic of the SMURF 3-stage compressor rig. R1, R2, R3 represent Rotor 1, 2 and 3, and S1, S2, S3 represent Stator 1, 2 and 3

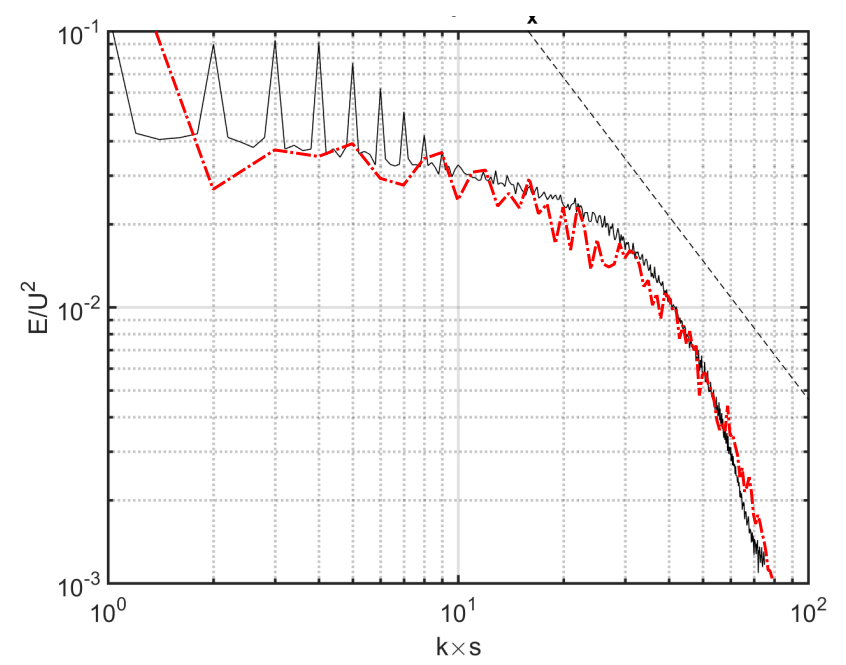

FIGURE 12. Comparison of the predicted turbulence spectrum (red) at $25 \%$ axial chord upstream of the stator leading-edge, and measured spectrum in the multi-stage rig at midspan Rotor 3 exit (black)

changes in midspan streamtube height from stator inlet to exit were measured to be around $3 \%$. The stator geometry at midspan was also essentially two-dimensional, and as such the simulations were run with both 2-dimensional geometry and boundary conditions. In order to ensure the inflow turbulence simulated in the simulations was representative of the rig conditions, a lengthscale and intensity were chosen to match experimental data obtained from hotwire measurements at the inlet to the stator within the multi-stage compressor test rig, which in this case were $1 \%$ of chord and $T u=3.5 \%$ respectively. A comparison of the measured turbulence spectrum and the spectrum from the simulations is shown in Figure 12; the spectrum is taken at a plane downstream of the inlet plane and $0.25 C_{a x}$ upstream of the stator leading-edge. The peaks in the measured spectrum are due to the blade-passing and harmonics and are therefore not captured in the simulations. In terms of the non-rotor-resolved unsteadiness, the results show that the prescribed spectrum matches closely the experimental measurement and thus the simulated turbulence represents reasonably well the multi-stage environment albeit in the absence of wakes.

A comparison of the total-pressure wake profile measured at Stator 3 exit and the prediction (case Re3E5) is shown in Figure 13. In the free-stream the experimental data shows the redistribution of total pressure across the passage due to the effects of upstream wakes. Since there are no rotor wakes in the com-

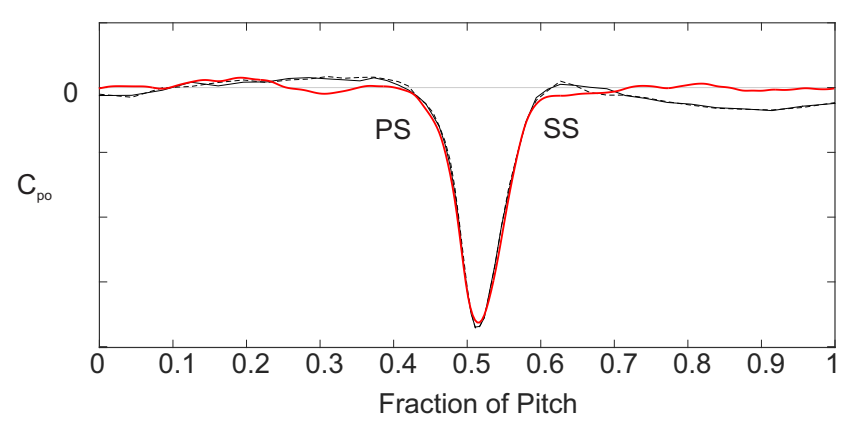

FIGURE 13. Comparison of predicted total pressure wake profile (red) with experimental measurement measured in the multi-stage rig (black)
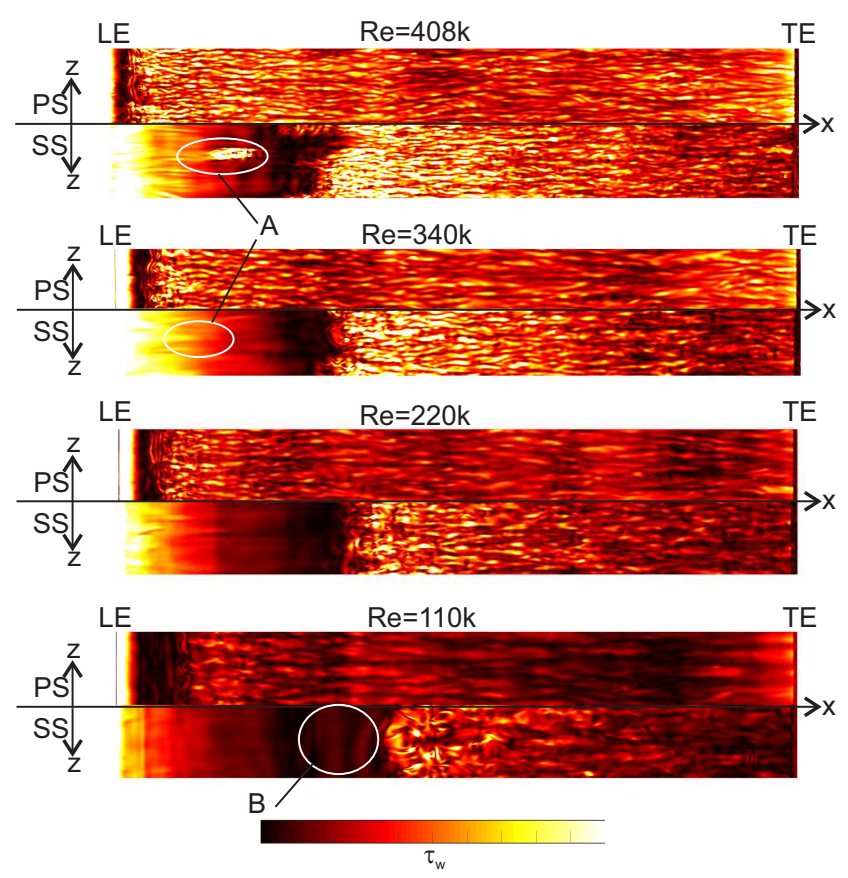

FIGURE 14. Instantaneous wall shear stress on the suction surface (SS) and pressure surface (PS)

putational simulations, the total pressure outside of the wakes in relatively uniform. Within the wake both the computational and experimental data match closely, both in terms of the wake depth and width; the computed wake depth and width are around $1.5 \%$ shallower and $3.5 \%$ wider than the experiment respectively. Although rotor wakes are not included in the simulation, this comparison indicates that the loss and mixing of the wake from the simulation to a large extent captures that observed in a representative multi-stage environment, despite the absence of wakes.

\section{RESULTS \\ Flow structure}

Instantaneous wall shear-stress plots showing the growth of instabilities in the boundary layer are shown Figure 14. Klebanoff streaks (labelled A) can be observed upstream of the transition point. The intensity of these streaks appears to reduce as the Reynolds number is reduced. Streaks are also observed 
downstream of the transition regions on both pressure and suction surfaces, although again the intensity of these streaks reduces with Reynolds number. Reducing Reynolds number also gives rise to separated-flow transition and the emergence of twodimensional Kelvin-Helmholtz roll-ups (labelled B).

The unsteady flow structures on the suction-surface are observed in Figure 15. The figure shows that in general both two-dimensional and three-dimensional instabilities occur within the transition region; spanwise roll-ups develop in the separated shear-layer (an example of which is labelled B), and these are followed by three-dimensional breakdown to turbulence. The development of two-dimensional instabilities clearly moves downstream as the Reynolds number is reduced. The threedimensional breakdown to turbulence leads to the formation of hair-pin structures (labelled C) as widely reported in previous studies of turbulent boundary layers (see for instance $[15,16]$ ). These grow in size as the Reynolds number is reduced. The size of these hair-pin structures is expected to determine the length of the non-equilibrium region, since the time-scale over which these structures transfer energy to smaller scales will be determined by their length-scale. The next section determines the length of the non-equilibrium region using the time-average results from the simulations.

\section{Size of non-equilibrium regions}

The simulations can be used to calculate the percentage of the suction surface boundary layer which exhibits nonequilibrium behaviour. This is achieved by determining the integrated turbulence production across the boundary layer $\mathrm{Pr}$ according to Equation 2:

$$
\operatorname{Pr}=\frac{1}{U_{e}^{2}} \int_{0}^{\delta} \overline{u_{i} u_{j}} \frac{\partial U_{i}}{\partial x_{j}} d y
$$

In order to illustrate how the region of non-equilibrium is determined, Figure 16 shows the variation of suction surface $\mathrm{Pr}$ with axial distance for $R e=340 k$ and a snapshot of the instantaneous wall shear stress in the region of peak turbulence production. We choose the start of the turbulent non-equilibrium region as the point of peak $\mathrm{Pr}$; since the focus of this paper is on the turbulent non-equilibrium region we do not include the region of transition upstream of this point in the estimation of non-equilibrium length. Downstream of the point of peak $\mathrm{Pr}$, the flow is essentially fully turbulent and there is no indication of intermittent laminar regions which are observed in the wall shear stress upstream of the peak production point. The end point of the non-equilibrium region is determined as the point where $\operatorname{Pr}$ is within $2 \%$ of the far downstream value (in this case at $90 \% C_{a x}$ ).

The turbulence production for all the Reynolds number tested is shown in the top of Figure 17. The non-equilibrium region determined by the method described above is indicated on the plots by the shaded regions. The figure shows that nonequilibrium regions affect a significant portion of the blade surface: around $15 \%$ of the suction surface at $R e=408 k$, increasing to $35 \%$ of the suction surface at $R e=110 \mathrm{k}$. The non-equilibrium wetted area is of a similar size to the laminar wetted area, and in
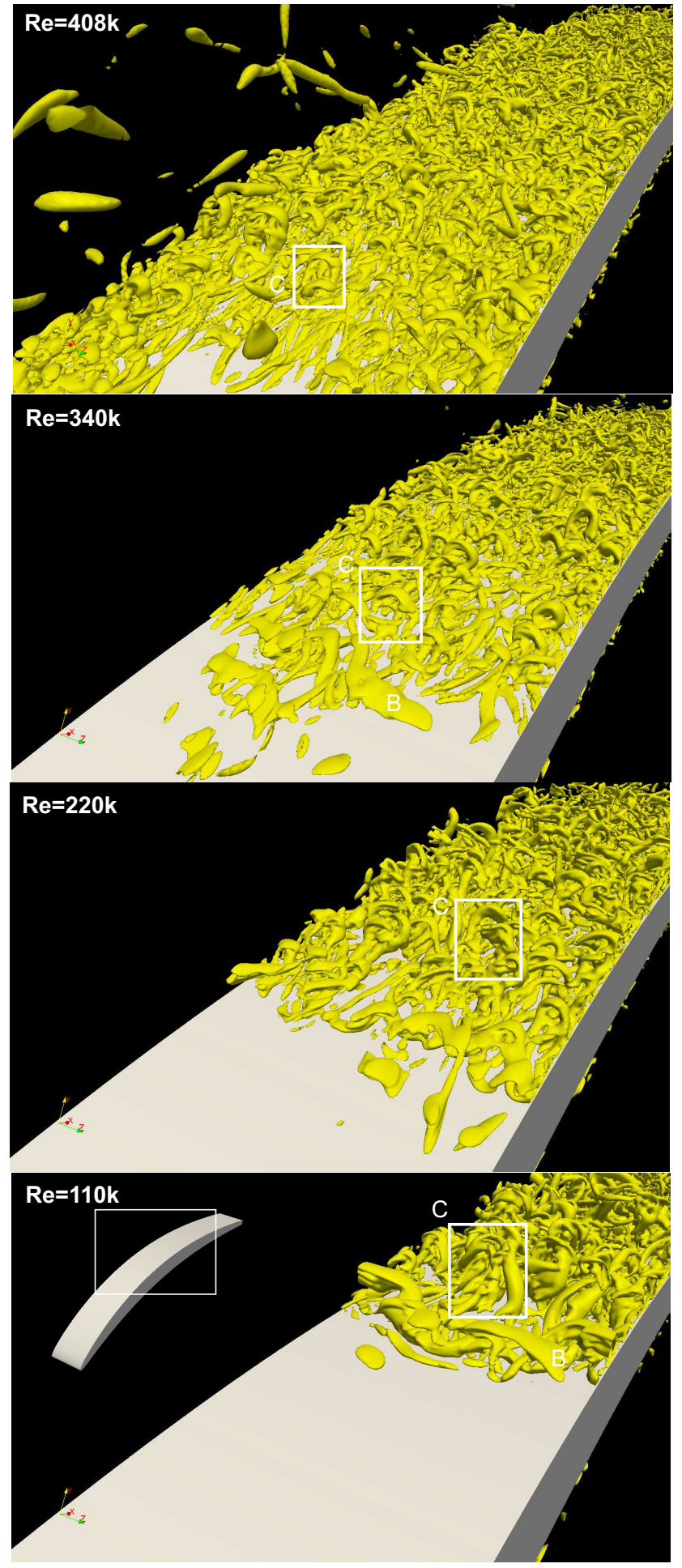

FIGURE 15. Suction-surface flow structure indicated by iso-surfaces of Q-criterion $=3 \times 10^{8} s^{-2} .(T u=3.5 \%)$

this case both reduce with Reynolds number by a similar proportion.

The lower part of Figure 17 shows the regions where the turbulence production and dissipation are out of balance. These regions are determined from the advection of turbulent kinetic energy $\vec{V} k$ assuming turbulent diffusion is small; contours indicating regions where the production rate either significantly 


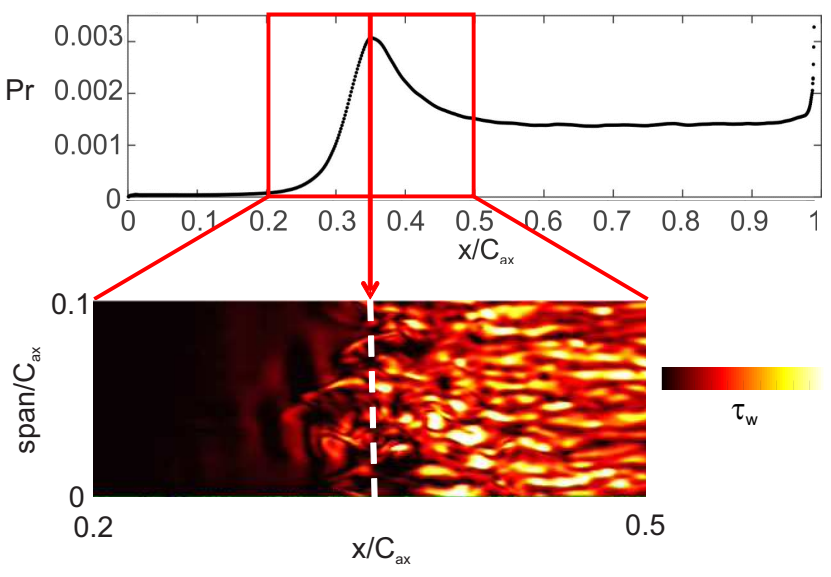

FIGURE 16. Suction surface turbulence production (top) and instantaneous wall shear stress $(R e=340 k, T u=3.5 \%)$

exceeds the turbulent dissipation rate (i.e., $\nabla \cdot(\vec{V} k)>>0)$ are shown in red, and contours where the dissipation is significantly larger than the production rate $(\nabla \cdot(\vec{V} k)<<0)$ are shown in blue. The figure shows that the dissipation lags significantly behind the production of turbulence. In the region of transition the production spreads from near the wall to the outer boundary-layer, while the dissipation growth lags the production and remains confined to the near-wall flow. Both these regions of imbalance increase in size as the Reynolds number is reduced and this is consistent with the increase in length of the non-equilibrium region observed above as well as the growth in size of the hair-pin structures observed in the instantaneous flow field (see Figure 15).

The next section introduces a reduced-order model in order to determine how these non-equilibrium effects influence performance.

\section{REDUCED-ORDER MODELLING}

In order to determine how non-equilibrium turbulence affects loss a method which de-couples the effects of transition and non-equilibrium behaviour is required. Here we use an industrystandard reduced order model in order to do this. The reducedorder model commonly used in industry to predict profile loss is MISES. MISES is a steady 2D/quasi-3D Euler code with a coupled boundary-layer solver. For the current work, 2D calculations were performed, where the boundary conditions were set to match the iLES/DNS simulations.

In the following sections we first compare the loss prediction from MISES with Reynolds number with the iLES/DNS data to verify that MISES gives the correct variation in loss with Reynolds number. Then we compare the turbulence production from MISES with the iLES/DNS to determine the veracity of the non-equilibrium model in MISES.

\section{Transition point modelling}

For the current investigation the suction surface and pressure surface transition points are prescribed in MISES at a point to match the transition point observed in the iLES/DNS simulations. As explained above, this was estimated from the point of peak turbulence production $\operatorname{Pr}$ (see Figure 16). Comparisons

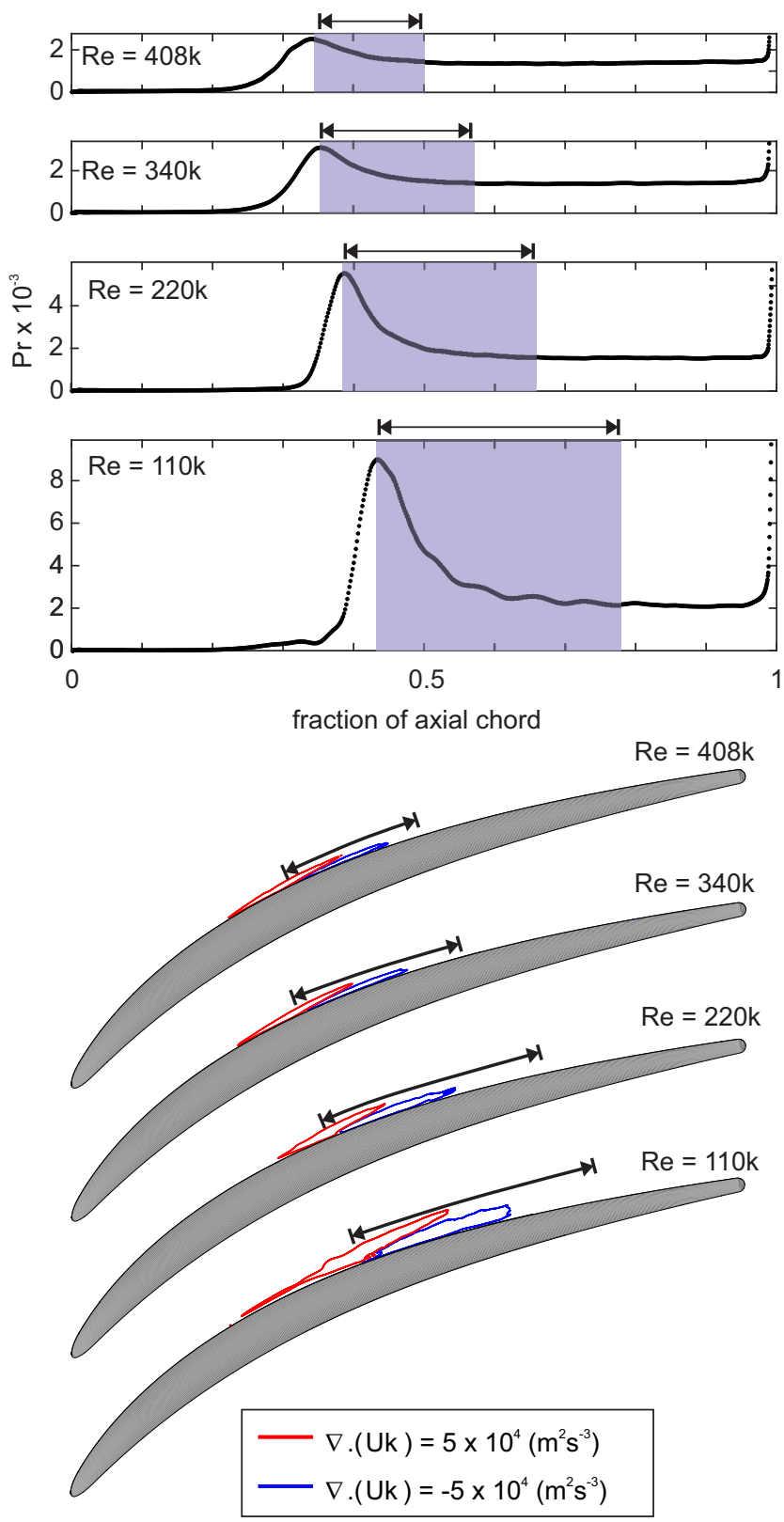

FIGURE 17. Turbulent kinetic energy production and divergence of turbulent kinetic energy flux in the region of suction-surface transition.
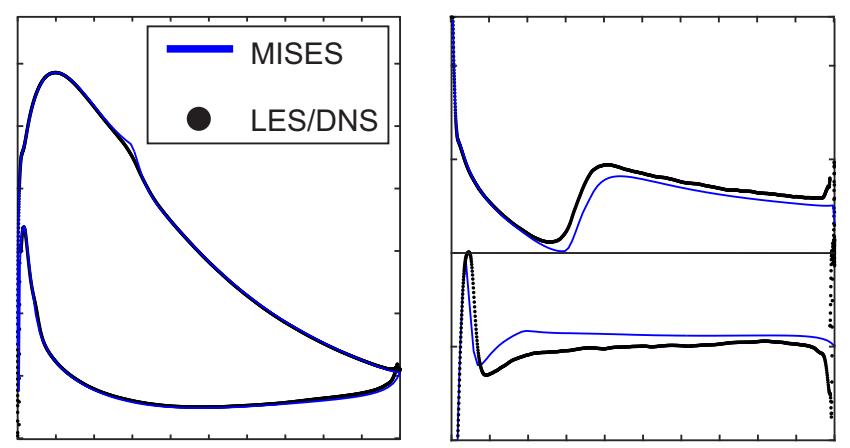

FIGURE 18. Loading and skin friction comparison with MISES with prescribed transition $(R e=340 k)$ 


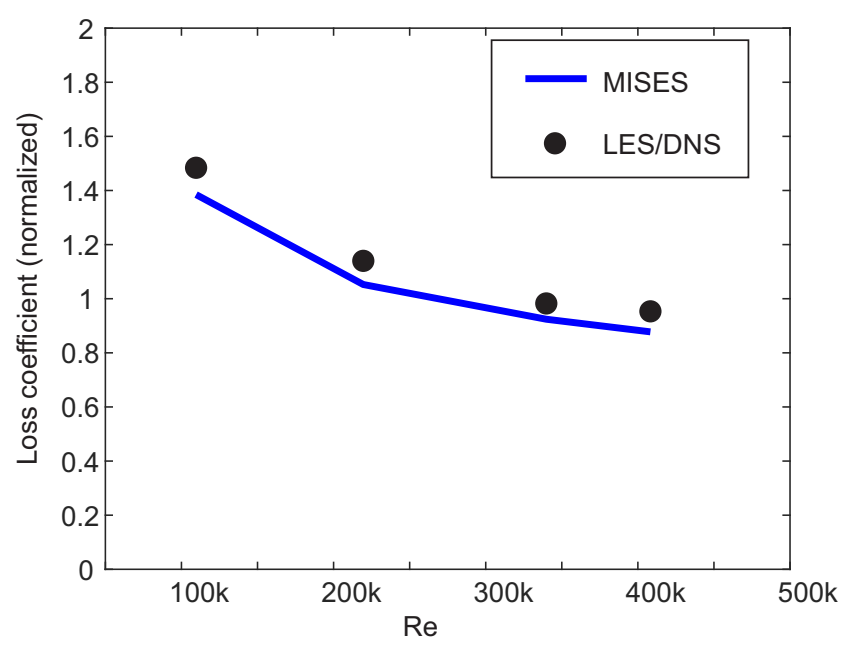

FIGURE 19. Loss prediction comparison with MISES with prescribed transition

of the predicted loading and skin-friction from MISES and the iLES/DNS are shown in Figure 18. The results show that this method of prescribing transition achieves a close match in both loading and skin friction.

A comparison of the loss variation with Reynolds number is shown in Figure 19. This figure shows that MISES tends to predict a loss around 5\% lower than the high-fidelity simulations, while the variation in loss with Reynolds number is in close agreement with the iLES/DNS. The exit flow angle predicted by MISES (not shown here) was also in close agreement with the simulations, being within $0.03 \mathrm{deg}$ for all cases tested.

The results verify the ability for MISES to predict performance when transition is prescribed to match the high-fidelity simulations.

In the next section we verify the accuracy of the nonequilibrium model in MISES as discussed next.

\section{Non-equilibrium modelling}

In the turbulent boundary layer the dissipation coefficient, and therefore loss, is determined in MISES by the skin friction coefficient $c_{f}$ and the Reynolds stress coefficient $c_{\tau}=$ $\left(\overline{u^{\prime} v^{\prime}} / u_{e}\right)_{\max }$ :

$$
c_{d}=\frac{c_{f}}{2} U_{s}+c_{\tau}\left(1-U_{s}\right)
$$

where $U_{s}$ is an effective normalized slip velocity determined from the local shape factor and momentum thickness Reynolds number $R e_{\theta}$. The first term on the right hand side of the equation represents the dissipation due to the time-average strain within the boundary layer, while the second term on the right accounts for the conversion of kinetic energy from the base flow into turbulent kinetic energy; this is the shear work done by the turbulent stresses in the boundary layer.

The approach in MISES is to assume that the skin friction $c_{f}$ responds immediately to the change in boundary layer state at transition, while the turbulent shear $c_{\tau}$ responds according to a

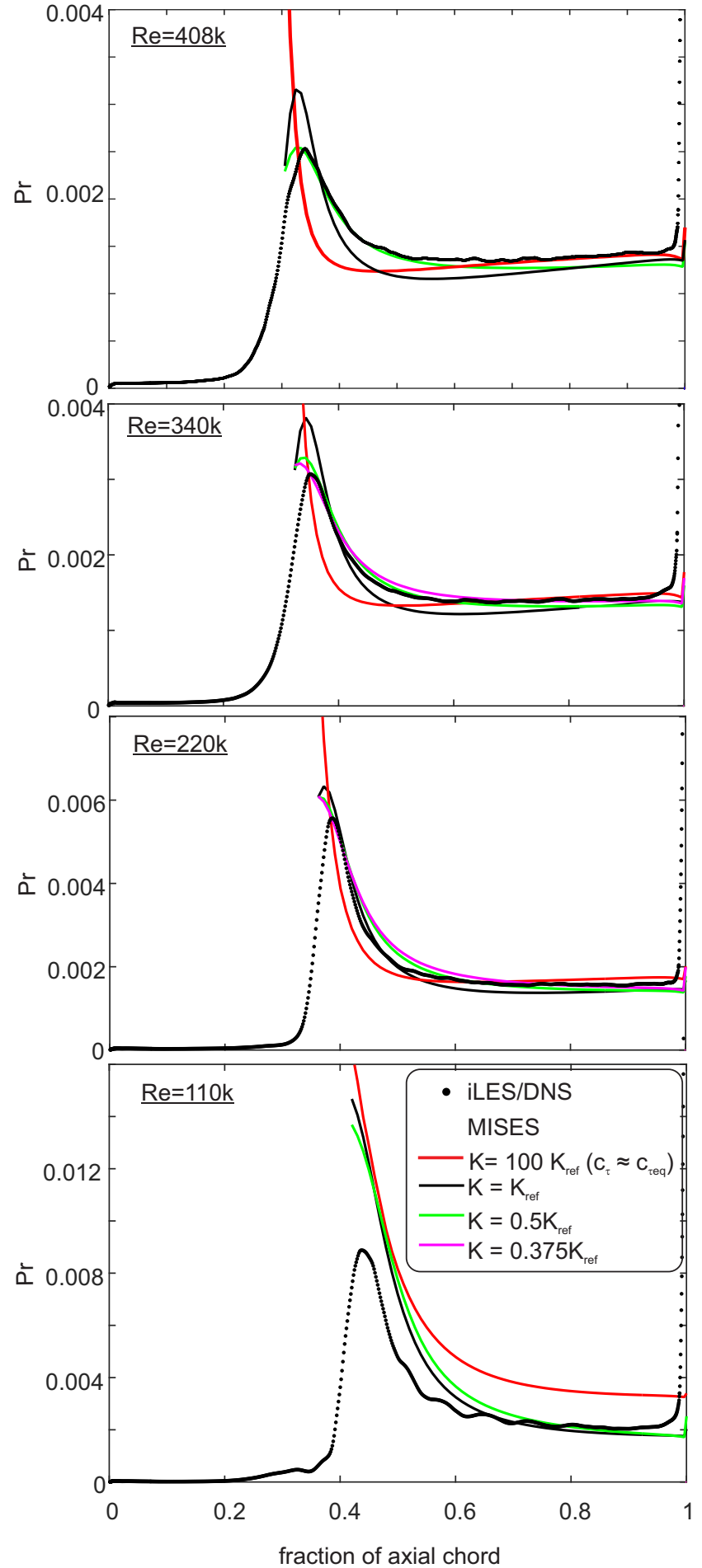

FIGURE 20. The effect of shear-lag constant $K$ on turbulence production as compared to iLES/DNS.

shear-lag model determined by Equation 4:

$$
\frac{\delta}{c_{\tau}} \frac{d c_{\tau}}{d s}=K\left(\sqrt{c_{\tau e q}}-\sqrt{c_{\tau}}\right)
$$

where $K$ is the lag constant. The actual $c_{\tau}$ lags the local equilibrium value $c_{\tau e q}$ which is derived from the $G-\beta$ turbulent equi- 


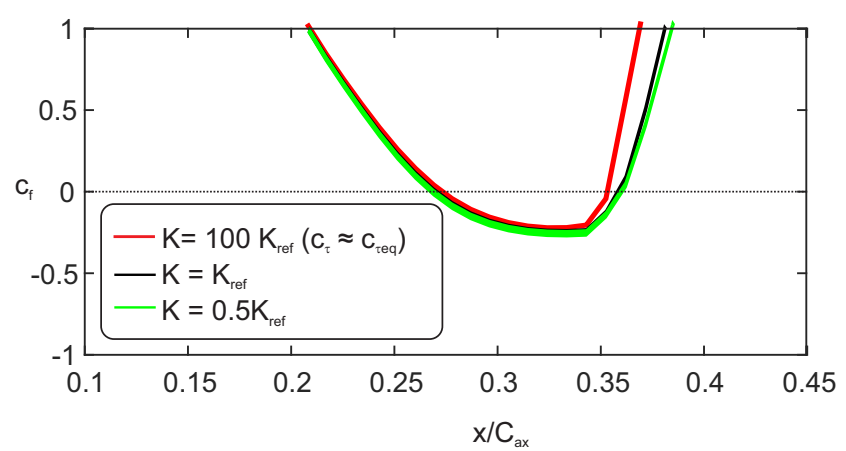

FIGURE 21. Skin friction in region of suction-surface separation bubble $(R e=220 k)$.

librium locus [7]. MISES only uses the shear-lag equation downstream of transition; upstream of transition laminar correlations are used to determine the dissipation and $c_{\tau}$ is not used. This is essentially a transport equation for turbulent shear. In order to solve Equation 4 , the value for $c_{\tau}$ at transition is required, which MISES determines from the following correlation:

$$
c_{\tau}=c_{\tau e q} \times 3.24 \exp \left(\frac{-6.6}{H-1}\right) .
$$

The accuracy of the shear-lag model can be assessed by comparing the MISES turbulence production term $\operatorname{Pr}=c_{\tau}\left(1-U_{s}\right)$ with the turbulent production within the boundary layer predicted by the DNS (see Equation 2)

The turbulent production from the iLES/DNS and the reduced order model are shown in Figure 20. The reduced order model has been run with four values of lag constant $K$. These are the reference value used by default in MISES $K=K_{\text {ref }}=5.6$, and values $K=0.5 K_{\text {ref }}, 0.375 K_{\text {ref }}$ and $K=100 K_{\text {ref }}$. Cases where $K=0.5 K_{\text {ref }}$ and $0.375 K_{\text {ref }}$ are cases where equilibrium behaviour is delayed. $K=100 K_{\text {ref }}$ ensures near equilibrium behaviour. For this case, Equation 5 was modified so that the transition point value of $c_{\tau}$ was set to the equilibrium value. It should be noted that because the boundary layer development is modified by changes in the shear lag, this also modifies $c_{\tau e q}$, and therefore downstream of the transition point the values of $c_{\tau e q}$ in the 'equilibrium' case depart from the $c_{\tau e q}$ values for the other non-equilibrium cases.

For the three highest Reynolds numbers $(R e=220 k, 340 k$ and $408 k$ ) the shear-lag model is observed to accurately predict the non-equilibrium behaviour (see Figure 20). The values of $K$ which give the closest agreement with the DNS are $K=0.5 K_{\text {ref }}$ and $0.375 K_{r e f}$. For the lowest Reynolds $(R e=110 K)$ the shearlag model was found to substantially overestimate the initial turbulence production after transition. At $R e=110 \mathrm{~K}$ it was not possible to obtain data when $K=0.375 K_{\text {ref }}$ due to the development of large separations which gave rise to poor convergence. In this Reynolds number regime bubble sizes become large and this shear-lag model is unlikely to perform well in this case. It is interesting to note that despite its simplicity, this shear-lag model performs remarkably well in terms of capturing the correct turbulence production.
The results show that as $K$ is reduced, and therefore nonequilibrium effects are increased, there is a reduction in the peak turbulence production this is important because this reduces the subsequent dissipation and therefore loss (as discussed later). The reduction in turbulence production was also found to affect the size of separation bubbles. Figure 21 shows the skin friction in the region of separation for $R e=220 k$. The figure shows that as the lag constant is reduced, the point of reattachment shifts downstream and the bubble length increases. The skin friction downstream of the bubble is also modified. The reduction in turbulence production as $K$ is reduced delays reattachment meaning that a thicker boundary layer is generated further downstream when reattachment does eventually occur.

\section{Effect of non-equilibrium boundary layers on loss}

The results so far indicate that non-equilibrium boundary layer behaviour can affect profile loss in two ways. First, nonequilibrium behaviour of attached turbulent boundary layers can lower profile loss by locally reducing the dissipation coefficient of the boundary layer. Secondly, non-equilibrium behaviour of separated boundary layers can delay reattachment and thus increase the bubble size thus increasing loss. In this section we make use of the reduced-order model to determine the effect of non-equilibrium boundary layers on loss as suction surface transition position is varied.

Figure 22 shows the variation of loss at a range of Reynolds numbers $R e=110 K-1 M$ using MISES with prescribed transition points from close to the leading-edge (where the flow is attached) to further downstream to where separation occurs (as indicated on the figure). For each Reynolds number line, the loss has been determined at a range of prescribed transition points and shear-lag constants. Results are shown for a range of lag constants, to indicate how non-equilibrium effects modify the loss. As above, data for $K=100 K_{\text {ref }}$ indicates the loss which would occur if the boundary layer was in a near equilibrium state throughout the whole turbulent part of the surface. The figure shows that in general non-equilibrium effects tend to reduce loss a part from in regions of separated flow transition.

Figure 23 shows contours of the percentage change in loss between equilibrium cases $K=100 K_{\text {ref }}$ and cases with $K=$ $0.375 K_{\text {ref }}$ to indicate the potential affect of non-equilibrium boundary layers on loss. The figure shows that in regions of attached flow, non-equilibrium effects reduce loss by as much as $8 \%$. The benefit results directly from the lower suction surface loss because non-equilibrium effects reduce turbulence shear (production) within the boundary layer. As transition moves downstream, and the flow moves from attached to separated flow this behaviour reverses and equilibrium effects increase loss by up to $6 \%$. The delay in turbulence production now tends to increase loss, particularly at high Reynolds numbers where bubble sizes are small; in this case the delay in production leads to a delay in the bubble closure and thus higher bubble losses as shown earlier. The overall variation in loss which could occur due to non-equilibrium effects is $14 \%$ moving from attached to separated flow transition.

It is interesting to note that in general non-equilibrium effects tend to reduce loss. Also shown in Figure 23 is the variation 


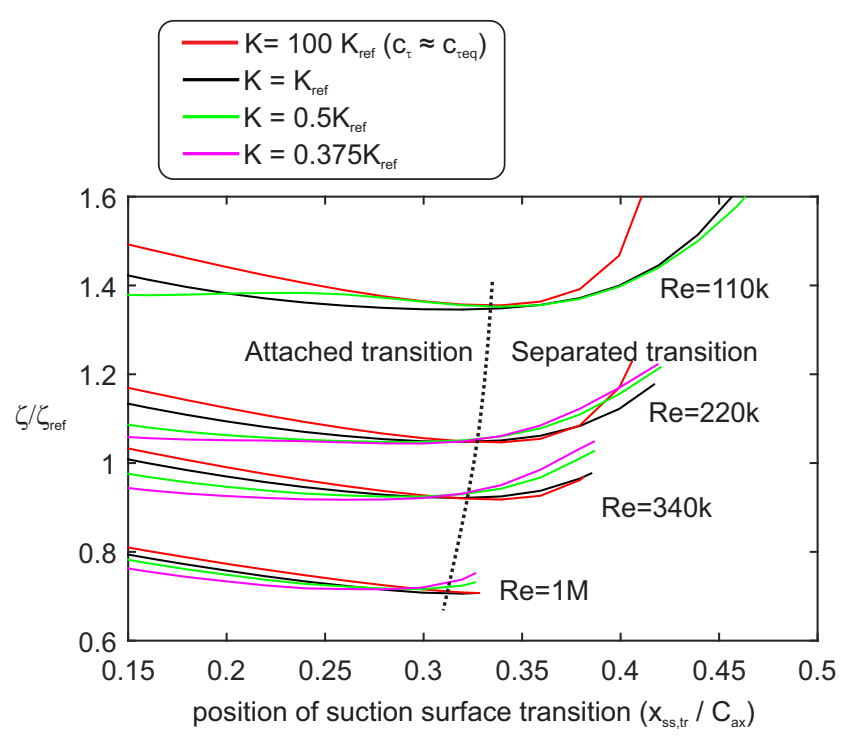

FIGURE 22. The effect of transition point and lag-constant $K$ on suction surface boundary layer loss as predicted by MISES.

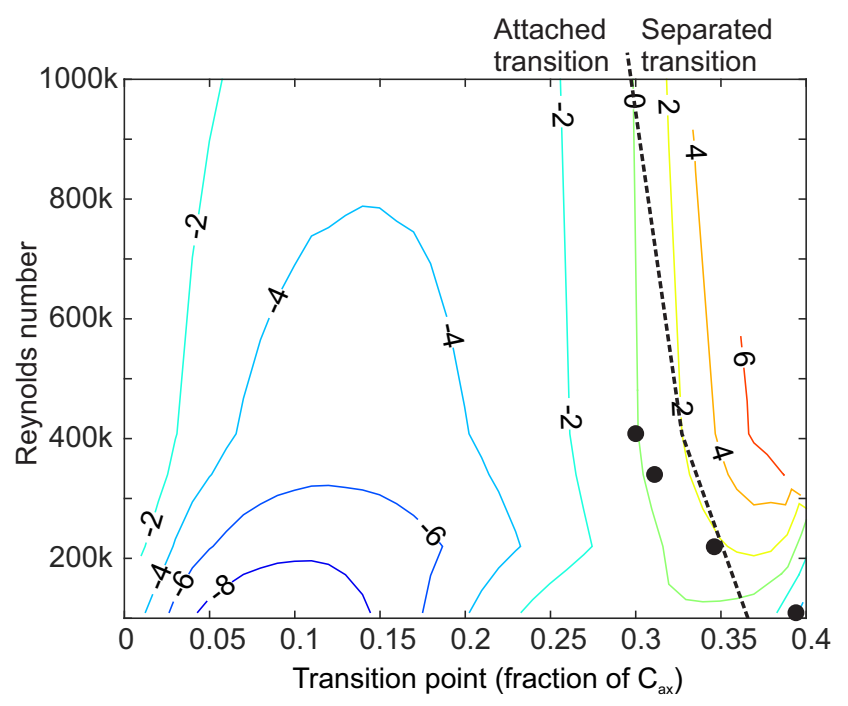

FIGURE 23. Predicted percentage change in suction-surface loss due to non-equilibrium boundary layers. iLES/DNS data indicated by the black dots.

in transition location determined from the iLES/DNS data; these show as Reynolds number is increased the compressor blade moves into a region where non-equilibrium tends to reduce loss. This may lead one to speculate whether one can exploit this benefit? This may be achievable through different loading distributions, surface roughness or by exploiting the effect of incoming wakes, for instance.

\section{CONCLUSIONS}

The paper addressed three questions. The first of these was to determine the extent to which non-equilibrium turbulence affects compressor boundary layers. The results show that for the Reynolds numbers tested in the paper $(R e=110-408 k)$, nonequilibrium flows affected between $15-35 \%$ of the suction sur- face, which was comparable to the regions of laminar wetted area for a given Reynolds number.

The second question was to determine if current reducedorder models capture non-equilibrium behaviour. We found that if the model constant was tuned to match the iLES/DNS, the shear-lag model used in MISES did broadly predict the correct turbulence production, albeit with a value of lag constant around half of the current recommended value.

Finally, using this model we then determined the effects on loss of non-equilibrium turbulence. The results indicated that non-equilibrium effects generally reduce loss apart from in regions where small separation bubbles occur. For attached-flow transition, non-equilibrium effects tend to reduce peak turbulence production thus reducing losses by as much as $8 \%$. For separated-flow transition, provided bubble sizes are small, nonequilibrium effects delay reattachment and thus increase losses by upto $6 \%$. The overall variation in loss which could occur due to non-equilibrium effects is $14 \%$ moving from attached to separated flow transition.

The paper shows that a significant region of the compressor boundary layer is affected by non-equilibrium turbulence, and that this behaviour offers the possibility of significantly changing profile loss. This raises the prospect of exploiting nonequilibrium effects to reduce loss.

\section{ACKNOWLEDGMENT}

The authors gratefully acknowledge the support of RollsRoyce plc who funded the computational costs for this work. The data given in Figure 2 was provided by Pawel J. Przytarski and his input is gratefully acknowledged.

\section{REFERENCES}

[1] Cumpsty, N. A., 1989. Compressor Aerodynamics. Longman Scientific \& Technical.

[2] Denton, J. D., 1993. "Loss mechanisms in turbomachines". ASME Journal of Turbomachinery, 115, pp. 621-656.

[3] Mayle, R. E., 1991. "The role of laminar-turbulent transition in gas turbine engines". ASME Journal of Turbomachinery, 113, pp. 509-537.

[4] Walker, G. J., 1993. "The role of laminar-turbulent transition in gas turbine engines a discussion". ASME Journal of Turbomachinery, 115, pp. 207-218.

[5] Schreiber, H. A., Steinert, W., and Kusters, B., 2002. "Effects of reynolds number and free-stream turbulence on boundary-layer transition in a compressor cascade". ASME Journal of Turbomachinery, 124, pp. 1-9.

[6] Hughes, J. D., and Walker, G. J., 2001. "Natural transition phenomena on an axial compressor blade". ASME Journal of Turbomachinery, 123, pp. 392-401.

[7] Green, J., Weeks, D., and Brooman, J., 1977. "Prediction of turbulent boundary layers and wakes in compressible flow by a lag-entrainment method". $R$ M Report 3791,Aeronautical Research Council, HMSO, London.

[8] Drela, M., 2014. Flight Vehicle Aerodynamics. MIT Press.

[9] DeBonis, J., 2013. "Solutions of the Taylor-Green Vortex Problem Using High-Resolution Explicit Finite Differ- 
ence Methods". 51st AIAA Aerospace Sciences Meeting including the New Horizons Forum and Aerospace Exposition(February).

[10] Tam, C. K. W., and Webb, J. C., 1993. "Dispersionrelation-preserving finite difference schemes for computational acoustics". Journal of Computational Physics, 107, pp. 262-281.

[11] Poinsot, T. J., and Lele, S. K., 1992. "Boundary conditions for direct simulations of compressible viscous flows". Journal of Computational Physics, 101, pp. 104-129.

[12] Zaki, T. A., Wissink, J. G., Rodi, W., and Durbin, P. A., 2010. "Direct numerical simulations of transition in a compressor cascade: the influence of free-stream turbulence". Journal of Fluid Mechanics, 665, pp. 57-98.

[13] Yao, Y., Thomas, T., and Sandham, N. D., 2001. "Direct numerical simulation of turbulent flow over a rectangular trailingedge". Theoretical and Computational Fluid Dynamics, pp. 337-358.

[14] Phillips, L., and Fyfe, D., 2011. "Turbid: A routine for generating random turbulent inflow data". Naval Research Laboratory Report, NRL/MR/6040-11-9357.

[15] Robinson, S. K., 1991. "Coherent motions in the turbulent boundary layer". Annual Review of Fluid Mechanics, 23.

[16] Adrian, R. J., 2007. "Hairpin vortex organization in wall turbulence". Physics of Fluids, 19. 